\title{
Flow behavior in weakly permeable micro-tube with varying viscosity near the wall
}

\author{
Ravikant R. Gupta ${ }^{{ }^{*}}$, Vineet Kumar', Shri Chand ${ }^{1}$ \\ ${ }^{1}$ Indian Institute of Technology Roorkee, Department of Chemical Engineering, Roorkee 247667, India \\ ${ }^{2}$ Indian School of Mines, Department of Chemical Engineering, Dhanbad 826004, India \\ "Corresponding author: e-mail: ravikant4u7@gmail.com
}

\begin{abstract}
Weakly permeable micro-tubes are employed in many applications involving heat and/or mass transfer. During these processes, either solute concentration builds up (mass transfer) or steep change in temperature (heat transfer) takes place near the permeable wall causing a change in the viscosity of the fluid. Results of the present work suggest that such change in viscosity leads to a considerable alteration in the flow behavior, and the commonly assumed parabolic velocity profile no longer exists. To solve the problem numerically, the equation of motion was simplified to represent permeation of incompressible, Newtonian fluid with changing viscosity through a micro-tube. Even after considerable simplification, the accuracy of the results was the same as that obtained by previously reported results for some specific cases using rigorous formulation. The algorithm developed in the present work is found to be numerically robust and simple so that it can be easily integrated with other simulations.
\end{abstract}

Keywords: hollow-fiber, micro-tube, permeation, algorithm, variable viscosity, velocity profile, equation of motion, navier-stokes equation.

\section{INTRODUCTION}

Laminar flow through porous tubes has been studied since a long time ${ }^{1}$. The internal laminar flow with fluid injection or suction at the porous wall has been investigated extensively for many engineering applications, including the design and analysis of nuclear reactors, combustion chambers, food-drying processes, heat pipes, solar air collectors, fuel cell and purification processes by reverse osmosis and ultrafiltration ${ }^{2}$. In all such applications, the viscosity of the flowing fluid inside the tube no longer remains constant either due to the concentration polarization or due to the temperature gradient. However, in almost all theoretical investigations, the viscosity of flowing fluid has been assumed constant (particularly while solving the equation of motion) for the sake of simpler model equations ${ }^{3-4}$. In the present work, the two-dimensional steady-state equation of motion has been simplified in terms of constant density and low radial velocity with varying local viscosity to predict the velocity profile numerically.

Although, many CFD (computational fluid dynamic) based software are readily available for investigating the flow through porous tubes under various conditions ${ }^{5-7}$, many times these software work as a black-box and takes abnormally longer time to converge. Considering this, in the present work, a simplified, fast, and accurate numerical tool has been developed that can be used for peeping into the experimental survey to estimate behaviors of any parameter quantitatively. These tools can also be used to study the effects of variation in the properties of fluids, and the effect of channel geometry in a flexible but defined way.

Classical Papers of Berman ${ }^{8}$, Yuan and Finkelstein', and Kozinski et al. ${ }^{10}$ are the basis of most of these CFD based analyses of flow through porous tube or duct. These authors presented analytical solutions based on perturbation treatment of fully developed laminar flow with the parabolic velocity profile in rectangular and tubular channels (assuming constant fluid properties) by solving steady state two-dimensional Navier-Stokes equation (i.e., the equation of motion with constant density and constant viscosity) along with the equation of continuity. Also, these authors assumed that the radial velocity profile was invariant in the axial direction since magnitude of the permeate flow rate through the tube/channel wall $\left(Q_{\mathrm{w}}\right)$ is so small that it can be approximated by a constant average value all along the length. Correlations for velocity profiles obtained by these authors (or their extension) are widely used for the simulation of channels having small dimensions ${ }^{11-12}$. The major drawback of these formulations is that these cannot handle effects of the changing viscosity of flowing fluid due to the concentration polarization (in the case of mass transfer problems) or due to the temperature gradient (in heat transfer problems). Considering this, in the present work, the steady state equation of motion is being used for developing a numerical tool to predict velocity profile inside a tubular micro-channel with varying viscosity of the fluid.

\section{MODELING FLOW THROUGH TUBULAR HOLLOW- -FIBERS}

In cases of a hollow-fiber, the equation of motion can be expanded in the 2-D cylindrical coordinate system giving the $x$ - and $r$-component in terms of stress tensor as ${ }^{13}$ :

$\rho\left(\mathrm{u} \frac{\partial \mathrm{u}}{\partial \mathrm{x}}+\mathrm{v} \frac{\partial \mathrm{u}}{\partial \mathrm{r}}\right)=-\frac{\partial \mathrm{p}}{\partial \mathrm{x}}-\left(\frac{\partial \tau_{\mathrm{xx}}}{\partial \mathrm{x}}+\frac{1}{\mathrm{r}} \frac{\partial\left(\mathrm{r} \cdot \tau_{\mathrm{rx}}\right)}{\partial \mathrm{r}}\right)$

and

$\rho\left(\mathrm{u} \frac{\partial \mathrm{v}}{\partial \mathrm{x}}+\mathrm{v} \frac{\partial \mathrm{v}}{\partial \mathrm{r}}\right)=-\frac{\partial \mathrm{p}}{\partial \mathrm{r}}-\left\{\frac{\partial \tau_{\mathrm{xr}}}{\partial \mathrm{x}}+\frac{1}{\mathrm{r}} \frac{\partial\left(\mathrm{r} \cdot \tau_{\mathrm{rr}}\right.}{\partial \mathrm{r}}\right\}$

respectively. The continuity equation in expanded form becomes

$\frac{\partial \rho u}{\partial \mathrm{x}}+\frac{1}{\mathrm{r}} \frac{\partial(\mathrm{r} \cdot \rho \mathrm{v})}{\partial \mathrm{r}}=0$

With the known behavior of processes with weak permeation and some simplifying assumptions, equations (1a) and (1b) can be simplified to a great extent. In cases of 
constant value of permeation throughout the length of the tube, the rate of change of radial velocity in the axial direction, $\partial v / \partial x$, is zero. Also, due to the weak inward or outward flow of permeate, $v \partial v / \partial r$ is negligibly small. Therefore, both the terms on the left hand side of the Eq. (1b) can be neglected.

Further, since the radial velocity is small, fluid momentum in the radial direction (at a particular cross-section inside the tube) can be assumed to be insignificant compared to the axial momentum. This ultimately makes all terms on the right hand side of the Eq. (1b) insignificant indicating that pressure at a particular section of the permeating tube can be assumed to be constant (i.e., $\partial p / \partial r \approx 0)$ which supports the simplifying assumption used in many cases of weak permeation ${ }^{14}$. These indicate that for fluid dynamic studies inside a tubular channel with weak permeation, only the axial component of the momentum equation and the equation of continuity is sufficient.

Secondly, in Eq. (1a) representing axial force-momentum balance, the transfer of axial momentum in radial direction takes place in two ways (i) due to viscous shear force in the axial direction, and (ii) due to bulk movement (convection) of fluid in the radial direction. Because of the very small value of the radial velocity, it can be easily visualized that there is no appreciable contribution of this radial velocity towards the convective transfer of axial momentum in the radial direction as compared to the axial momentum transfer in the radial direction due to viscous forces (molecular momentum transfer). Thus, the second term on the left hand side of the Eq. (1a) becomes less significant. Further, in a tubular permeable tube of uniform cross-section, the rate of change of axial velocity in the axial direction, $\partial u / \partial x$ is expected to be negligibly small (since inward or outward permeation rate is several orders of magnitude less than the axial flow rate). Thus both the terms on the left hand side of the Eq. (1a) vanishes. Furthermore, for an incompressible fluid flowing in a tube of uniform cross-section, the normal stress $\tau_{x x}$ becomes zero. With these, the equation of motion (Eq. 1a) for a weakly permeable tube (with a uniform cross-sectional area and incompressible fluid) reduces to:

$\frac{\partial \mathrm{p}}{\partial \mathrm{x}}=-\frac{\partial\left(\tau_{\mathrm{rx}}\right)}{\partial \mathrm{r}}-\frac{\tau_{\mathrm{rx}}}{\mathrm{r}}$

By making a force balance on the volume element of radius $r$ (Fig. 1), the shear stress $\tau_{r x}$ is given by:

$\tau_{\mathrm{rx}}=\lim _{\Delta \mathrm{x} \rightarrow 0}\left(\frac{\pi \mathrm{r}^{2} \Delta \mathrm{P}}{2 \pi \mathrm{r} \Delta \mathrm{x}}\right)=-\frac{\mathrm{r}}{2} \frac{\partial \mathrm{p}}{\partial \mathrm{x}}$

After differentiating Eq. (4) with respect to $r$, and substituting $\partial p / \partial r=0$, we get

$-\frac{\partial\left(\tau_{\mathrm{rx}}\right)}{\partial \mathrm{r}}=\frac{1}{2} \frac{\partial \mathrm{p}}{\partial \mathrm{x}}$

Substituting Eq. (5) in the Eq. (3) and rearranging we get the greatly simplified equation of motion:

$\frac{\partial \mathrm{p}}{\partial \mathrm{x}}=\frac{2 \tau_{\mathrm{rx}}}{\mathrm{r}}$

Equation (6) is the basis of the well-known HagenPoiseuille equation in which fluid viscosity is taken as constant. One cannot integrate Eq. (6) unless viscosity

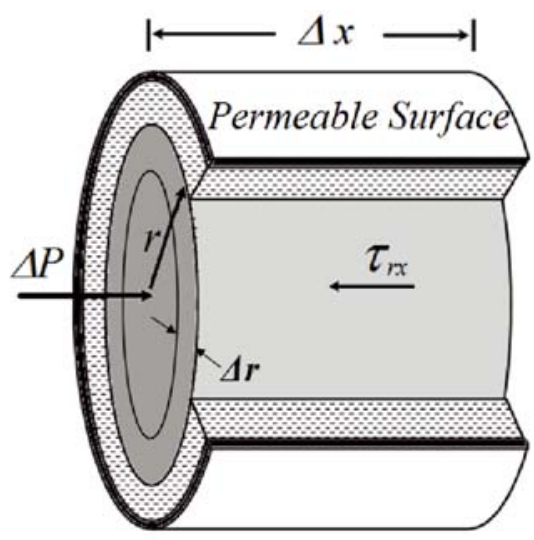

Figure 1. Pressure and shear forces on an integral volume element of radius $r$ (shaded cylindrical element) inside a tube section of length $\Delta x$, with a differential volume element of thickness $\Delta r$

as a function of radial position is known. In most of the practical cases of flow with fluid injection or suction through the porous wall, the viscosity of flowing fluid does not remain constant either due to concentration polarization or due to changing temperature, which themselves depend on velocity profile. This makes it difficult to solve the equation even numerically.

On the other hand, if viscosity is constant, HagenPoiseuille equation is applicable even in the case of flow through a circular tube with permeation, provided $Q_{x}$ is constant (or at least nearly constant). Thus, in case of very small $\Delta x$ (Fig. 1), i.e., only in a small length segment of the micro-tub, it can be assumed that there is no appreciable change in axial volumetric flow rate $\left(Q_{x}\right)$ of liquid within this element, and there exists an average value of viscosity, $\mu_{\text {eff }}$ (applicable only to this volume element), that can be used in the Hagen-Poiseuille equation such that

$\mathrm{Q}_{\mathrm{x}}=\frac{\pi \mathrm{R}^{4}}{8 \mu_{\mathrm{eff}}} \frac{\partial \mathrm{p}}{\partial \mathrm{x}}$

where $Q_{x}$ can be taken as the overall volumetric flow rate of fluid entering the micro-tube section shown in Figure 1 , and $\mu_{\text {eff }}$ is the effective viscosity of the fluid that can be determined as follows.

Substituting $\partial p / \partial x$ from the above Eq. (7) into Eq. (6) and introducing shear stress in terms of Newton's law of viscosity and rearranging we get:

$\frac{\partial \mathrm{u}}{\partial \mathrm{r}}=-\frac{4 \mathrm{Q}_{\mathrm{x}} \mathrm{r} \mu_{\mathrm{eff}}}{\pi \mu_{\mathrm{r}} \mathrm{R}^{4}}$

The above equation can be solved with the boundary condition $u(x, R)=0$ within one axial volume element of width $\Delta x$ (Fig. 1) giving:

$\mu(\mathrm{r}) \frac{\partial \mathrm{u}}{\partial \mathrm{r}}=\frac{\pi \mathrm{r}^{2} \Delta \mathrm{P}}{2 \pi \mathrm{r} \Delta \mathrm{x}}$

Upon integration of Eq. (9) from the wall of the tube $(r=R ; u=0)$ to a distance $r$ from the center where the axial velocity $u$ is to be evaluated, gives:

$\mathrm{u}=\frac{\Delta \mathrm{P}}{2 \Delta \mathrm{x}} \int_{\mathrm{r}}^{\mathrm{R}} \frac{\mathrm{r}}{\mu(\mathrm{r})} \mathrm{dr}$

Now, the overall volumetric flow rate of the fluid can be obtained by integrating Eq. (10) as follows: 
$\mathrm{Q}_{\mathrm{x}}=\int_{0}^{\mathrm{R}} \mathrm{u} \cdot 2 \pi \mathrm{r} d \mathrm{r}=\frac{\pi \Delta \mathrm{P}}{\Delta \mathrm{x}} \int_{0}^{\mathrm{R}}\left[\int_{\mathrm{r}}^{\mathrm{R}} \frac{\mathrm{r}}{\mu(\mathrm{r})} \mathrm{dr}\right] \cdot \mathrm{rdr}$

Substituting Eq. (7) and rearranging we get

$\mu_{\mathrm{eff}}=\frac{\mathrm{R}^{4}}{8 \int_{0}^{\mathrm{R}}\left[\int_{\mathrm{r}}^{\mathrm{R}} \frac{\mathrm{r}}{\mu(\mathrm{r})} \mathrm{dr}\right] \cdot \mathrm{r} \mathrm{dr}}$

In a practical situation, however, the viscosity of a fluid depends on the local conditions of temperature and composition; therefore, a suitable function representing the variation of viscosity in terms of $r$ that can be used for integrating Eq. (12) may not be known. In such cases, the integral in the denominator of Eq. (12) can be obtained numerically in terms of as a function of local conditions using the following numerical integration based on the trapezoidal rule.

$\mu_{\text {eff }}=\frac{\mathrm{R}^{4}}{8 \sum_{\mathrm{j}=1}^{\mathrm{N}-1}\left[\left\{\sum_{\mathrm{m}=\mathrm{j}+1}^{\mathrm{N}}\left(\frac{\left(\mathrm{r}_{\mathrm{m}}-\Delta \mathrm{r} / 2\right) \Delta \mathrm{r}}{\mu\left(\mathrm{r}_{\mathrm{m}}-\Delta \mathrm{r} / 2\right)}\right)\right\} \mathrm{r}_{\mathrm{j}} \Delta \mathrm{r}\right]}$

Where, $N$ is the total number of integral (cylindrical) volume elements of nominal radius $r_{j}(j=1,2, \ldots N)$ as shown in Figure 1, and is the local viscosity of the fluid at the centre of a differential volume element bounded between radii $r_{m-1}$ and $r_{m}$.

Thus the simplified momentum equation (Eq. 6) and the equation of continuity (Eq. 2) are required to be solved for the CFD analysis a micro-tube, subject to the conditions that:

(i) The flowing fluid is Newtonian,

(ii) Fluid is incompressible, (iii) Wall permeation is weak,

(iv) Wall permeability is constant along the length,

(v) Tube length, $\Delta \mathrm{x}$, is small enough so that $2 \cdot \pi \cdot R \cdot \Delta \mathrm{x} \cdot$ $v_{w} / Q_{\text {in }}<<1$,

(vi) No-slip condition at the tube wall,

(vii) Cross-sectional area of the tube is uniform throughout,

(viii) Considering these, a long micro-tube of length $L$ can be assumed to be made of small elements of length $\Delta \mathrm{x}$ such that conditions (iv) and (v) are satisfied. Solution for the entire tube length can be achieved sequentially, starting from the inlet. A brief discussion on the algorithm that can be adopted to solve these equations sequentially is presented in Table 1 .

\section{RESULTS AND DISCUSSION}

To validate the outcome of the proposed algorithm, a micro-tube of radius $(R) 0.0007 \mathrm{~m}$, length $(L) 0.2 \mathrm{~m}$, permeation velocity $\left(v_{\mathrm{w}}\right) 0.0001 \mathrm{~m} / \mathrm{s}$ and feed velocity $\left(u_{\mathrm{o}}\right.$ at $\left.x=0\right)$ of $0.5 \mathrm{~m} / \mathrm{s}$ is assumed. The viscosity of the feed, $\mu_{0}$ is taken as 0.001 Pa.s.

To execute the algorithm, the micro-tube is discretized in the cylindrical volume elements of thickness $\Delta \mathrm{r}$ and length $\Delta \mathrm{x}$ such that $2 \cdot \pi \cdot R \cdot \Delta x \cdot v_{w} / Q_{i n} \leq 0.0001$. The grid size independency of the computer code (including Eq. 13 to estimate $\mu_{\text {eff }}$ ) was tested for constant viscosity $\mu(r)=\mu_{0}$ at all $r$. Several numbers of differential volume elements were tried; three of them $(N=25,100$, and $500)$ are presented in Figure 2. It is evident from the figure that for 100 and 500 numbers of differential volume elements, results are overlapping. This indicates that 100 differential volume elements in the radial direction are

Table 1. Solution algorithm

\begin{tabular}{|c|c|}
\hline Action & Remarks \\
\hline $\begin{array}{l}\text { Variables/constants: } \\
r_{j}, \Delta r, x, \Delta x, u_{i, j}, \mu_{i, j}, \rho_{i, j}, Q_{x}, \Delta q, N, R, L, v_{w}\end{array}$ & $\begin{array}{l}N \text { is the number of differential volume elements in the radial direction such that } r_{N}=R \text {, and } \\
\text { variables such as } u_{i, j}, \mu_{i, j} \text {, and } \rho_{i, j} \text { are the values at the centre of the differential volume } \\
\text { element between } r_{j-1} \text { and } r_{j}\end{array}$ \\
\hline Input: $\quad Q_{x}, R, L, \Delta x, v_{w}, \mu_{0}$ etc. & $\begin{array}{l}\text { Feed flow rate }\left(\mathrm{m}^{3} / \mathrm{s}\right) \text {, radius }(\mathrm{m}) \text {, total tube length }(\mathrm{m}) \text {, axial volume element, permeation } \\
\text { velocity }(\mathrm{m} / \mathrm{s}) \text {, feed viscosity (Pa.s), etc. }\end{array}$ \\
\hline \multicolumn{2}{|l|}{ Initialize: $x=\Delta x / 2$ and $i=1 ; \Delta r=R / N, \mathrm{r}_{\mathrm{j}}=\mathrm{j} \Delta \mathrm{x}$} \\
\hline \\
\hline \multicolumn{2}{|l|}{ Estimating: $\mu_{j}, \mu_{\text {eff }}$ and $\Delta P$} \\
\hline $\begin{array}{lr}\mu_{\mathrm{j}}=\mu_{0}\left(1+\beta\left(\frac{\mathrm{r}_{\mathrm{j}}}{\mathrm{R}}\right)^{\mathrm{n}}\right) & \text { (assumed } \\
\text { correlation, otherwise } \mu_{\mathrm{j}}=\mathrm{f}\left(\mathrm{T}_{\mathrm{j}}, \mathrm{P}_{\mathrm{j}}, \mathrm{C}_{\mathrm{j}}\right)\end{array}$ & We used $\beta$ and $n$ to adjust magnitude and steepness of the viscosity variation \\
\hline$\mu_{e f f}=\frac{R^{4}}{8\left(\begin{array}{l}N-1 \\
j=1\end{array}\left\{\begin{array}{l}N \\
m=j+1\end{array}\left(\frac{\left(r_{m^{-}} r / 2\right) \Delta r}{\mu\left(r_{m}-\Delta r / 2\right)}\right)\right\} r_{j} \Delta r\right)}$ & Equation (13) \\
\hline$\Delta P=8 Q_{x} \mu_{\text {eff }} \Delta x /\left(\pi R^{4}\right)$ & Equation (7) \\
\hline \multicolumn{2}{|c|}{ Calculate $u_{i, j}$ usin g equation of motion (equation 6) and Newton's law of viscosity (equation 9): } \\
\hline$T_{j}=\Delta P r_{j} /(2 \Delta x)$ & For all $j(=1,2, \ldots, N)$ \\
\hline$u_{i, N}=T_{N}(\Delta r / 2) / \mu_{N}$ & For $j=N$ with no-slip condition near the wall, using equation (15) \\
\hline$u_{i, j}=u_{i, j+1}+T_{j} \Delta r / \mu_{j}$ & for $j=N-1, N-2, \ldots, 2,1$ \\
\hline \multicolumn{2}{|c|}{ Calculate $v_{j}$ using eq uation of continuity (equation 2 ): } \\
\hline$\Delta q=\left(\rho_{i, j} u_{i, j}-\rho_{i-1, j} u_{i-1, j}\right)\left(2 \pi r_{j-1 / 2} \Delta r\right)$ & For all $j(=N, N-1, \ldots, 2) ; \quad 2 \pi r_{j-1 / 2} \Delta r=\pi\left(r_{j}^{2}-r_{j-1}^{2}\right)$ \\
\hline$v_{N}=v_{w}$ & For $j=N$ \\
\hline \multirow[t]{2}{*}{$v_{j-1}=\left(v_{j} \rho_{i, j} 2 \pi r_{j} \Delta x+\Delta q\right) /\left(\rho_{i, j-1} 2 \pi r_{j-1} \Delta x\right)$} & for $j=N-1, N-2, \ldots, 2$ \\
\hline & In case of single component incompressible fluid, density $\rho_{i, j}=\rho_{m, n}=$ constant \\
\hline \multicolumn{2}{|l|}{ Increment in $x$} \\
\hline \multicolumn{2}{|l|}{$i=i+1$} \\
\hline \multicolumn{2}{|l|}{$x=x+\Delta x$} \\
\hline \multicolumn{2}{|l|}{$Q_{x}=Q_{x}-2 \pi R \Delta x v_{w}$} \\
\hline \multicolumn{2}{|l|}{ If $(x<L)$ go to :Loop $(i)$} \\
\hline End. & (End of Table 1) \\
\hline
\end{tabular}




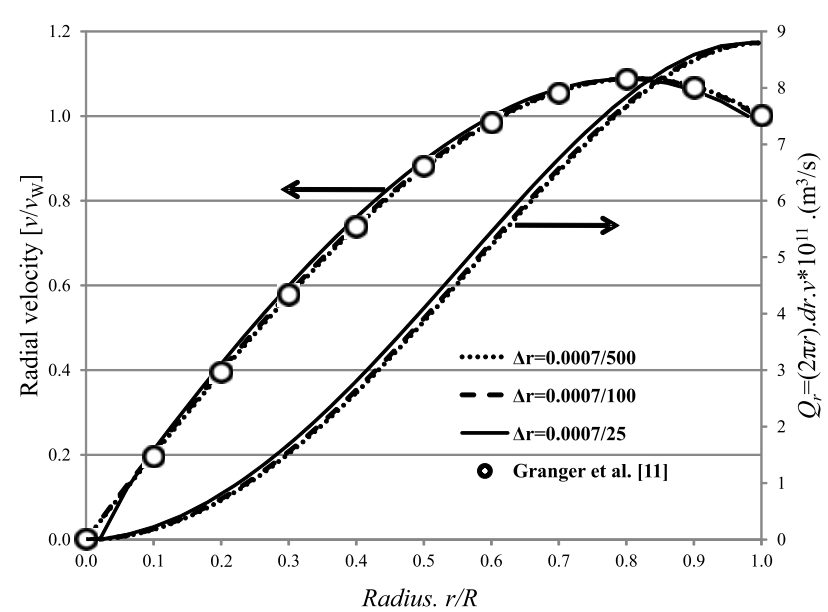

Figure 2. Radial velocity, and radial flow inside a micro-tube of radius $R(=0.0007 \mathrm{~m})$, for different size of volume element $\Delta r(=\mathrm{R} / \mathrm{N}$; where $\mathrm{N}$ is the number of volume element in radial direction 25, 100 and 500 , respectively)

sufficient for the present work. The results obtained by Granger et al..$^{15}$, with constant viscosity $\left(\mu(r)=\mu_{0}\right)$ are also compared in this graph. An excellent agreement between the present results (with $\mathrm{N} \geq 100$ ) and those of Granger et al..$^{15}$ is an evidence of the fact that the terms (such as radial component of the equation of motion, $\partial p / \partial r, v \partial v / \partial r, \partial v / \partial x$, etc.) which were ignored using simplifying assumptions has no significant effect on the velocity components and these terms can be ignored safely.

To study the effect of changing viscosity of the fluid, three types of viscosity variation were considered. In the first case, viscosity increases sharply near the wall and remains almost constant in the core of flowing fluid. This case is similar to ultrafiltration and reverse osmosis in which viscosity near the wall may be many orders of magnitude higher than the bulk viscosity of the fluid. The other two cases resemble permeation with heat transfer, where the change in viscosity may be less severe, but extends deeper inside the core of the flowing fluid (Fig. 3a). In reality, such viscosity variations are caused by the variation in concentration or temperature which can be predicted by introducing more terms in model equation representing heat and mass transfer. However, to avoid further complexity, and to see the effect of change in viscosity on the flow characteristics, the viscosity of fluid as a function of radial position is defined directly by assuming following hypothetical equation:

$\mu_{\mathrm{j}}=\mu_{0}\left[1+\beta\left(\frac{\mathrm{r}_{\mathrm{j}}}{\mathrm{R}}\right)^{\mathrm{n}}\right]$

A steep increase in viscosity near the tube wall is obtained by substituting $\beta=20$ and $n=32$ in equation (14), whereas the moderate increase or decrease in viscosity is predicted by putting $\beta=5$ and -0.7 , and $n=7$ and 4 , respectively (Fig. 3a).

In all three cases of viscosity variation, permeation velocity, $v_{w}$, is taken as constant all along the tube length (irrespective of viscosity of the fluid near the permeable surface). The resultant velocity profiles obtained by solving the model equations using the algorithm shown in Table 1 are interesting. In Fig. 3, the $x$-axis represents dimensionless radial distance measured from the centre (a)
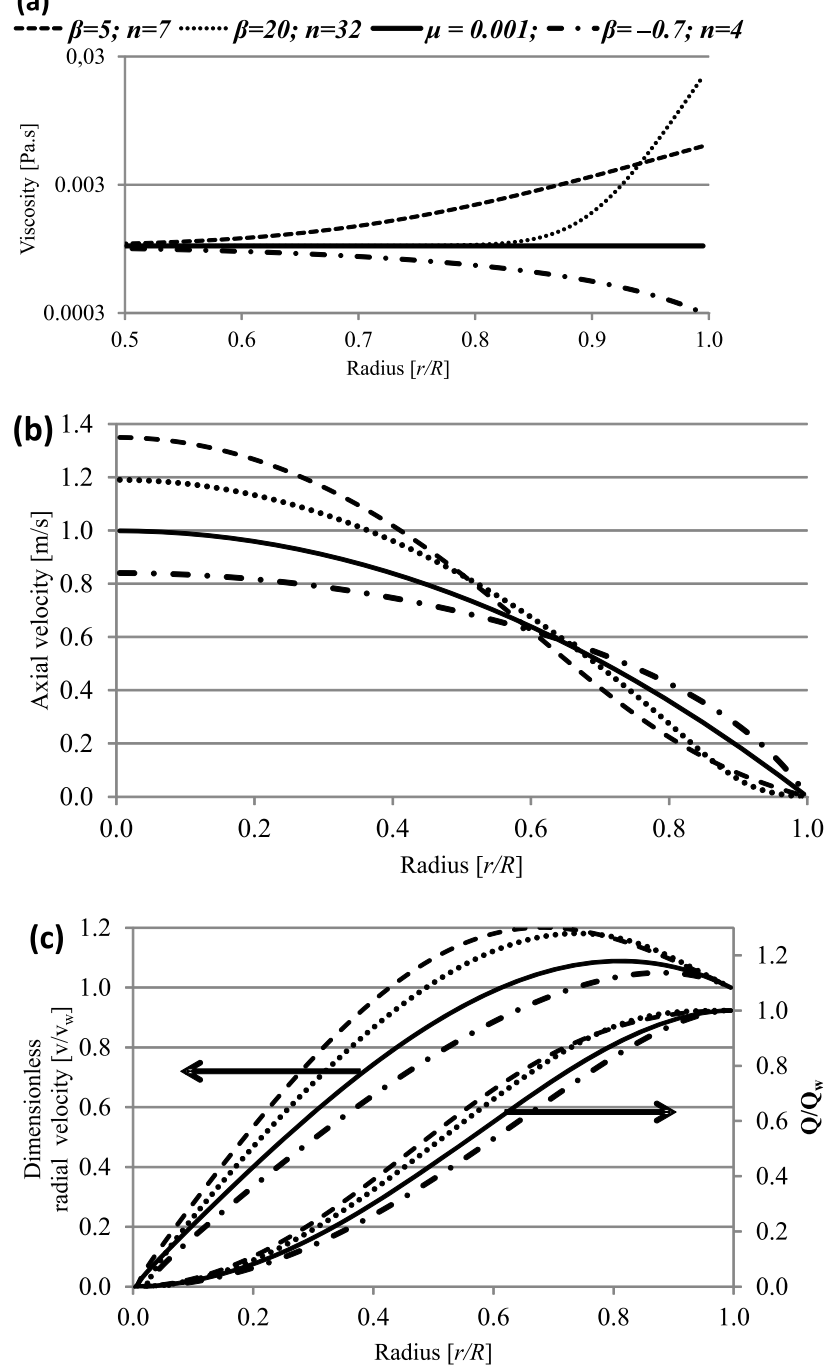

Figure 3. Assumed viscosity variation inside the micro-tube (a), Effect of change in viscosity on axial velocity profile (b), and dimensionless radial velocity and dimensionless volumetric flow rate (c).

of the tube. The variation in axial velocity is shown in Figure $3 \mathrm{~b}$. Dimensionless radial velocity $\left(v / v_{w}\right)$ and the dimensionless radial volumetric flow rate $\left(Q / Q_{w}\right)$ are plotted on the primary and secondary vertical axis of Figure 3c, respectively. The curves for the case of constant viscosity are represented by the solid line.

Evidently, the velocity profiles in case of variable viscosity (dashed, dotted and dash-dot lines) are significantly different from the condition when the viscosity is constant at (solid line). Also, the parabolic velocity profile, which is assumed in most of the theoretical analysis, cannot be applied in processes where change in viscosity of the fluid is expected. It is also interesting to note that even in the case in which viscosity change is restricted in a region close to the wall (dotted line in Figure 3 representing steep change in viscosity), its effect on the axial velocity extends from tube wall to center of the tube.

As a result of increased viscosity near the permeable surface, tangential velocity, $u$, is significantly less than that predicted by a parabolic profile. Similarly, in the case of decreasing viscosity (say due to increase in temperature), the tangential velocity near the wall is higher than expected, however, in the central core, velocity is significantly low. This, in most of the cases, will lead to an adverse effect on heat and mass transfer. In cases of 
severe change is viscosity, one may expect the situation to become even worse.

It is also evident that the effect of a change in viscosity on the flow characteristics is more in the case when the change in viscosity extends deeper inside the tube. Although a sharp change in viscosity near the wall has a lesser influence on flow characteristics, but the velocity gradient near the permeating surface decreases considerably. This may trigger a series of other events that may not favor permeation based operations.

\section{CONCLUSIONS}

Huge simplification in the equation of motion is possible without compromising with the accuracy of the results in case of the laminar flow of incompressible Newtonian fluid inside a weakly permeating micro-tube of uniform cross-section. Due to dropping off of the less significant terms, the simplified equation could handle some extra parameter, such as variable viscosity of the fluid, and the model could predict velocities in more complex situations. Although the double integration to evaluate effective viscosity is difficult to solve analytically, the trapezoidal rule based integration is sufficient for practical purposes. The algorithm developed in the present work is robust and simple so that it can be incorporated easily in other simulations. However, care must be taken to see the possibility of error getting accumulated while integrating using trapezoidal rule.

In order to incorporate the effect of variation in viscosity (either due to concentration polarization or due to temperature gradient) in a weakly permeable or impermeable tube, one can use the concept of effective viscosity being introduced in the present work through equation (12). For the case where integration of viscosity term is difficult, numerical integration using equation (13) can be performed.

The results indicate that the widely assumed parabolic velocity profile in a permeable micro-tube module is doubtful in cases when fluid properties are not constant and should be used with caution. Moreover, if fluid properties are constant, there is no need to solve the entire Navier-Stokes equation with radial pressure drop in cases of weak permeation

\section{ACKNOWLEDGEMENTS}

The authors gratefully acknowledge the financial and other related support from Indian Institute of Technology Roorkee.

\section{NOMENCLATURE}

$L \quad$ Length of the micro-tube (m)

$N \quad$ Number of volume element in radial direction $(-)$

$n \quad$ Constant exponent used in Equation 14

$P \quad$ Pressure $(\mathrm{kPa})$

$Q \quad$ Volumetric flow rate in the radial direction $\left(\mathrm{m}^{3} \mathrm{~s}^{-1}\right)$

$Q_{\text {in }} \quad$ Axial inlet volumetric flow rate of fluid across a cross-section of the tube $\left(\mathrm{m}^{3} \mathrm{~s}^{-1}\right)$

$Q_{\text {out }} \quad$ Axial exit volumetric flow rate of fluid across a cross-section of the tube $\left(\mathrm{m}^{3} \mathrm{~s}^{-1}\right)$
$Q_{x} \quad$ Axial volumetric flow rate of fluid at an axial position $x\left(\mathrm{~m}^{3} \mathrm{~s}^{-1}\right)$

$Q_{w} \quad$ Volumetric flow rate of the permeate through tube wall $\left(\mathrm{m}^{3} \mathrm{~s}^{-1}\right)$

$R \quad$ Radius of micro tube (m)

$r \quad$ Radial distance measured from the centre of the micro-tube $(\mathrm{m})$

$u \quad$ Local axial velocity $\left(\mathrm{m} \mathrm{s}^{-1}\right)$

$u_{\text {in }} \quad$ Average axial inlet velocity entering a volume element $\left(\mathrm{m} \mathrm{s}^{-1}\right)$

$u_{\text {out }} \quad$ Average axial inlet velocity exiting a volume element $\left(\mathrm{m} \mathrm{s}^{-1}\right)$

$v \quad$ Local radial velocity $\left(\mathrm{m} \mathrm{s}^{-1}\right)$

$V \quad$ Velocity vector $\left(\mathrm{m} \mathrm{s}^{-1}\right)$

$v_{w} \quad$ Permeation velocity at the tube wall $\left(\mathrm{m} \mathrm{s}^{-1}\right)$

$x \quad$ Axial distance measured from the inlet of the micro-tube $(\mathrm{m})$

$\beta \quad$ Constant used in Equation 14

$\Delta \mathrm{P} \quad$ Pressure difference $(\mathrm{kPa})$

$\Delta \mathrm{r} \quad$ Thickness of a differential volume element in radial direction $(\mathrm{m})$

$\Delta \mathrm{x} \quad$ Length of the volume element in axial direction (m)

$\mu_{0} \quad$ Viscosity of the fluid at the inlet conditions $(\mathrm{Pa} \cdot \mathrm{s})$

$\mu_{\text {eff }} \quad$ Effective viscosity of the fluid at a fixed axial position $(\mathrm{Pa} \cdot \mathrm{s})$

$\mu(r) \quad$ Fluid viscosity at a distance $r$ from the centre $(\mathrm{Pa} \cdot \mathrm{s})$

$\tau \quad$ Stress tensor $\left(\mathrm{N} \mathrm{m}^{-2}\right)$

$\tau_{r x} \quad$ Shear stress acting on $r$-face in $x$-direction $\left(\mathrm{N} \mathrm{m}^{-2}\right)$

$\tau_{x x} \quad$ Normal stress acting on $x$-face in $x$-direction $\left(\mathrm{N} \mathrm{m}^{-2}\right)$

\section{LITERATURE CITED}

1. Olson, F.C.W. (1949). Flow through a pipe with a porous wall. J. Appl. Mech. 16(1), 53-54.

2. Cheng, Y.C. \& Hwang, G.J. (1995). Experimental studies of laminar flow and heat transfer in a one-porous-wall square duct with wall injection. Int. J. Heat Mass Tran. 38(18), 3475-3484. DOI: 10.1016/0017-9310(95)00037-A.

3. Goosen, M.F.A., Sablani, S.S., Al-Hinai, H., Al-Obeidani, S., Al-Belushi, R. \& Jackson, D. (2005). Fouling of reverse osmosis and ultrafiltration membranes: a critical review. Separ. Sci. Technol. 39(10), 2261-2297. DOI: 10.1081/SS-120039343.

4. Xinhui, S., Liancun, Z., Xinxin, Z. \& Jianhong, Y. (2011). Homotopy analysis method for the heat transfer in a asymmetric porous channel with an expanding or contracting wall. Appl. Math. Model. 35(9), 4321-4329. DOI: 10.1016/j. apm.2011.03.009.

5. Ahmad, A.L., Lau, K.K., Bakar, M.A. \& Shukor, S.A. (2005). Integrated CFD simulation of concentration polarization in narrow membrane channel. Comput. Chem. Eng. 29(10), 2087-2095. DOI: 10.1016/j.compchemeng.2005.06.001.

6. Liang, Y.Y., Chapman, M.B., Weihs, G.F. \& Wiley, D.E. (2014). CFD modelling of electro-osmotic permeate flux enhancement on the feed side of a membrane module. J. Membr. Sci., 470, 378-388. DOI: 10.1016/j.memsci.2014.07.039.

7. Jun, C.L., Xiang J.Y. \& Dong, Hu Y. (2015). CFD simulations of the fluid flow behavior in a spacer-filled membrane module. Membr. Water Treat. 6(6), 513-524. DOI: 10.12989/ mwt.2015.6.6.513.

8. Berman, A.S. (1953). Laminar flow in channels with porous walls. J. Appl. Phys. 24(9), 1232-1235. DOI: 10.1063/1.1721476. 
9. Yuan, S.W. \& Finkelstein, A.B. (1956). Laminar pipe flow with injection and suction through a porous wall. Trans. ASME. 78, 719-724.

10. Kozinski, A.A., Schmidt, F.P. \& Lightfoot, E.N. (1970). Velocity Profiles in Porous-Walled Ducts. Ind. Eng. Chem. Fundam. 9(3), 502-505. DOI: 10.1021/i160035a033.

11. Tilton, N., Martinand, D., Serre, E. \& Lueptow, R.M. (2012). Incorporating Darcy's law for pure solvent flow through porous tubes: Asymptotic solution and numerical simulations. AIChE J. 58(7), 2030-2044. DOI: 10.1002/aic.13823.

12. Kim, A.S. \& Lee Y.T. (2011). Laminar flow with injection through a long dead-end cylindrical porous tube: Application to a hollow fiber membrane. AIChE J. 57(8), 1997-2006. DOI: 10.1002/aic.12430.

13. Bird, R.B., Stewart, W.E. \& Lightfoot, E.N. (2002). Transport phenomena ( ${ }^{\text {nd }}$ ed.). John Wiley \& Sons.

14. Vennela, N., Mondal, S., De, S. \& Bhattacharjee, S. (2012). Sherwood number in flow through parallel porous plates (Microchannel) due to pressure and electroosmotic flow. AIChE J. 58(6), 1693-1703. DOI: 10.1002/aic.12713.

15. Granger, J., Dodds, J. \& Midoux, N. (1989). Laminar flow in channels with porous walls. The Chem. Eng. J. 42(3), 193-204. DOI: 10.1063/1.1721476. 